\title{
New three-dimensional model based on finite element method of bone nanostructure: single TC molecule scale level
}

\author{
Tesnim Kraiem ${ }^{1}$, Abdelwahed Barkaoui ${ }^{1,2}$, Moez Chafra ${ }^{2}$, Ridha Hambli ${ }^{3}$, João Manuel \\ R. S. Tavares ${ }^{4}$
}

${ }^{1}$ Université de Tunis El Manar, Ecole Nationale d'Ingénieurs de Tunis, LR-11-ES19 Laboratoire de Mécanique Appliquée et Ingénierie (LR-MAI), 1002, Tunis, Tunisie

${ }^{2}$ Université de Tunis El Manar, Institut Préparatoire aux Etudes d'Ingénieurs d'El Manar, B.P 244, 2092, Tunis, Tunisie

${ }^{3}$ PRISME laboratory, EA4229, University of Orleans Polytech' Orléans, 8, Rue Léonard de Vinci 45072 Orléans, France

\footnotetext{
${ }^{4}$ Instituto de Ciência e Inovação em Engenharia Mecânica e Engenharia Industrial, Departamento de Engenharia Mecânioca, Faculdade de Engenharia, Universidade do Porto, Rua Dr. Roberto Frias, 4200-465 Porto, Portugal
}

\section{Corresponding author:}

Professor João Manuel R. S. Tavares

Departamento de Engenharia Mecânica

Faculdade de Engenharia da Universidade do Porto

Rua Dr. Roberto Frias

4200-465 Porto

Portugal

Phone: +351 225081 487, Fax: +351225081445

Email: tavares@fe.up.pt, url: www.fe.up.pt/ tavares 


\title{
New three-dimensional model based on finite element method of bone nanostructure: single TC molecule scale level
}

\begin{abstract}
At the macroscopic scale, the bone mechanical behavior (fracture, elastic) depends mainly on its components' nature at the nanoscopic scale (collagen, mineral). Thus, an understanding of the mechanical behavior of the elementary components is demanded to understand the phenomena that can be observed at the macroscopic scale. In this article, a new numerical model based on finite element method is proposed in order to describe the mechanical behavior of a single Tropocollagen molecule. Furthermore, a parametric study with different geometric properties covering the molecular composition and the rate hydration influence is presented. The proposed model has been tested under tensile loading. While focusing on the entropic response, the geometric parameter variation effect on the mechanical behavior of Tropocollagen molecule has been revealed using the model. Using numerical and experimental testing, the obtained numerical simulation results seem to be acceptable, showing a good agreement with those found in literature.
\end{abstract}

Keywords: Bone nanostructure; Tropocollagen; Alpha helix; Mechanical behavior. 


\section{Introduction}

The bone hierarchical structure allows a significant mechanical performance (elasticity, stiffness, ductility, energy dissipation and strength). Understanding and characterizing the bone properties are crucial in predicting diseases, such as osteoporosis, and even giving a targeted therapy in the advanced cases. The collagen molecule is observed at the lowest hierarchical level. Making up to 30\% of the body's protein content (Fratzl 2008), (Kadler et al. 2007), collagen is prevalent in blood vessels, tendons, cornea, skin, cartilage bone and teeth, and it has been identified in more than 27 types (described from I to XXVII) (van der Rest \& Garrone 1991) with 42 distinct polypeptide chains. Among these different types, type I collagen, which has a fibrillar structure and known as Tropocollagen (TC), is the most abundant one in the human body (Hulmes 2008). Ramachandran and Kartha (Ramachandran \& Kartha 2006) have made the first attempt to prove that the TC molecule, a right-handed helix, is composed by three polypeptide chains called alpha helices, each one is a left-handed helix. These three chains are held together by an interchain hydrogen bonds.

The alpha chains composing the TC molecule are almost identical. Each polypeptide chain is characterized by the repeat sequence Gly-X-Y, where Gly is a glycine exposed at the core of the protein, $\mathrm{X}$ and $\mathrm{Y}$ are amino acids (proline and hydroxyproline) exposed at its surface. The alpha helix has also a helical structure (Pauling \& Corey 1953) with a length of $300 \mathrm{~nm}$, a diameter of about $0.5 \mathrm{~nm}$ and a pitch of about $5.4 \mathrm{~nm}$.

Several analytical, experimental and computational studies have been conducted with the aim of predicting the mechanical behavior and properties of the TC molecule, which is considered as an anisotropic material (Saini \& Kumar 2015). However, most of the research was based on simplified models, considering that the molecule is isotropic and predicting only the Young's modulus $(E)$.

Using X-ray diffraction, Sasaki and Odajima (Sasaki \& Odajima 1996) examined the stressstrain relationship of a single TC molecule. A linear stress-strain behavior was observed even in static or dynamic applied tests. The Young's modulus $(E)$ resulting from a static test is slightly lower than that resulting from a dynamic test, revealing the viscous feature of the molecule.

Optical tweezers have been used by Sun and colleagues (Sun et al. 2002) in order to characterize the collagen mechanical behavior. The obtained force-extension curve was 
nonlinear, which was described by an inextensible worm-like chain model. In order to understand the mechanical behavior of the TC molecule and correlate the results with high resolution topography imaging, Bozec and Horton (Bozec \& Horton 2005) have used Atomic Force Microscopy (AFM). Two zones can be distinguished on the obtained load-deformation curve: the first corresponds to a toe region while the second corresponds to a linear one.

In the other hand, Loronzo and Caffarena (Lorenzo \& Caffarena 2005) executed a steered molecular dynamic simulation to determine mechanical properties of a single TC. For this purpose, (Pro-Hyp-Gly)4-Pro-Hyp-Ala-(Pro-Hyp-Gly)5 (Bella et al. 1994) was used as a TC alpha structure helix, at a temperature of $300{ }^{\circ} \mathrm{K}$ under 1 atm pressure. A tensile test was applied to the mass center of the N-terminal nitrogen atoms of a wet TC molecule along the longitudinal molecular axis, while the C-terminal was kept attached. The stretched molecule ( $2 \%$ of the molecular length) has a linear force-deformation curve. Similarly, the authors proved that most of the hydrogen bonds are responsible for the molecular stability as well as the coiling of the three alpha helices.

Using molecular dynamic method, Buehler (Buehler 2006) investigated the mechanical properties of the TC molecules under different types of loading consisting of tension, compression, bending and shear. The author aimed at the force field determination for the TC molecule sequence of (Pro-Hyp-Gly)4-Glu-Lys-Gly-(Pro-Hyp-Gly)5 (Bella et al. 1994). The force field is a mathematical expression giving the dependency relationship between the system energy and its particles' coordinates. There are two types of force fields: classical (MacKerell et al. 1998) and reactive (Van Duin et al. 2001). In the case of the classical force fields, the bonds between atoms are represented by harmonic springs, making it hard to crack the bonds and modeling the new ones. Not taking into account the chemical reactions is one of the main limitations of the classical force fields that the reactive one can exceed.

In 2008, Veld and Stevens (Veld \& Stevens 2008) carried out an atomistic simulation on a single TC molecule. The wet protein TC was pulled along its axis in order to determine its tensile strength and Young's modulus. Furthermore, two of the three helices were separated by pulling their $\mathrm{N}$-terminal residues in opposite directions perpendicular to the primary molecular axis. The separation was forced between the two first strands, while the third strand remained untouched. The internal strength of the molecule was then estimated.

Afterwards, Uzel \& Buehler (Uzel \& Buehler 2009) examined the effect of the amino acid motif variation on the mechanical properties of single TC collagen molecules, more precisely on the elastic deformation at different applied strains. A bottom-up computational 
materiomics approach, applied on four model sequence motifs found in type I collagen, illustrated the extreme influence of the amino acid motif variations on the TC molecule elastic behavior, prior to the softening or stiffening behavior. Uzel \& Buehler (Uzel \& Buehler 2009) were able to prove that the interpeptide interactions via $\mathrm{H}$-bonds vary widely with the motif type, attesting to its role in the molecule stability. These investigators approved the molecular inhomogeneous deformation hypothesis using direct stretching simulation of a TC segment that features the physiological amino acid sequence.

A simple structural model allowing to determine the viscoelastic properties of the TC molecule has been developed by Gautieri and co-authors (Gautieri et al. 2012). Using molecular simulations, the dependency of the viscosity and the Young's modulus on the applied stress has been proved.

The TC molecule is one of the main bone material components. However, the macroscopic mechanical behavior and properties of the bone material depend greatly on the properties and geometries of the different scales: microscopic (cortical and trabecular bone, osteon, lamella), nanoscopic (fiber, fibril (Vercher et al. 2014; Vercher-Martinez et al. 2015)) and microfibril (Barkaoui \& Hambli 2011; Barkaoui, Hambli, et al. 2014; Barkaoui, Chamekh, et al. 2014) as well as on the molecular compounds (mineral, collagen (TC molecules)).

According to previous numerical and experimental research, the crucial role and the great influence of the TC level scale has been revealed. Using the finite element method (FEM), the current work is a part of a multiscale modeling project, raging from nanoscale to macroscale. To our knowledge, no work has been carried out on the TC molecule using FEM. Hence, the aim of this paper was centered on three purposes: (i) proposing a new 3D FE model of a single TC molecule; (ii) validating the new FE model on the basis of experimental results, molecular dynamic simulation and theoretical model held on the TC molecule with the same geometric properties; and (iii) investigating the TC mechanical behavior by varying some structure parameters such as diameter and pitch, as well as the spacing between alpha helices of the TC molecule.

\section{Methods and tools}

In this study, FEM is used in order to analyze the mechanical behavior of a single TC molecule. For this purpose, a parametric 3D geometric model has been built. The FE model 
analysis allows to study the variation effect of the geometric parameters on the mechanical properties of the TC molecule.

\section{TC molecule geometry structure}

In the literature, several TC dimensions have been proved (Figure 1 and Table 1). This variability is linked to many factors. On the one hand, it depends on the used experimental setup such as the optical tweezers and the X-ray analysis, on the experimental conditions, on the applied method and the used parameters that fit the experimental data with the theoretical models. On the other hand, this variability depends on the molecule's hydration percentage, which was the aim of the studies carried out by Lees (Lees 1981) and Fratzl and collaborators (Fratzl et al. 1993) claiming that the dry TC molecule have a diameter of $1.09 \mathrm{~nm}$, while the wet TC molecule has a diameter of about 1.42 to $1.5 \mathrm{~nm}$.

It should be noted that the ends of the TC molecules are also non helical telopeptides $(\mathrm{N}$ and C telopeptides) (Kadler et al. 1996).

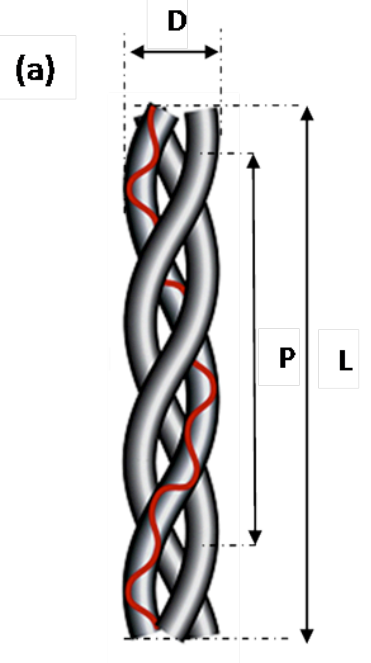

(b)

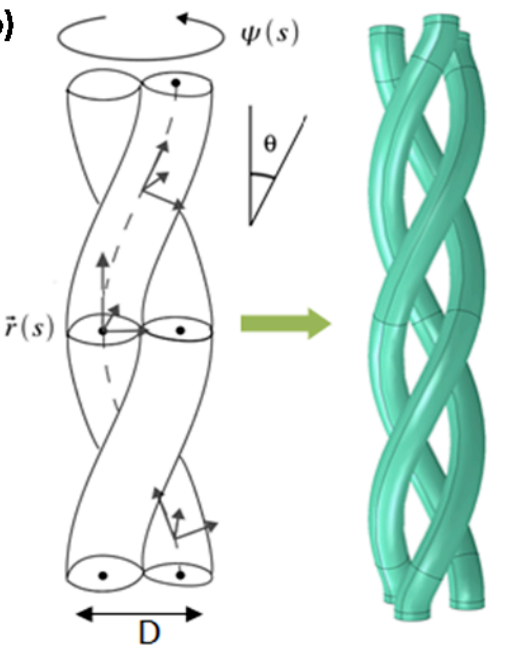

Figure 1: 3D geometric model generation: (a) parameters characterizing the TC molecule geometry ( $L$, $p, D)$; (b) (left) construction of the coiled coil mathematical model from the centerline equation, (right) generation of the geometric model of the TC molecule using a design software.

\section{Geometric model building}

The TC molecule is a right-handed helix composed by three alpha helices. Each one of the latter is a left-handed helix. In order to simplify the model, the alpha helix is considered as a 
cylinder following the shape of a major helix bran. The length $(L)$, the diameter $(d)$ and the pitch $(p)$ are the geometric dimensions of the TC major helix (Figure 1).

Table 1: The different TC molecule dimension values found in the literature.

\begin{tabular}{|c|c|c|}
\hline Parameter & Value & References \\
\hline \multirow{4}{*}{ Length $(L)$} & $287 \mathrm{~nm}$ & (Bozec \& Horton 2005) \\
\hline & $300 \mathrm{~nm}$ & $\begin{array}{l}\text { (Parry 1988), (Uzel \& Buehler } \\
\text { 2009), (Orgel et al. 2001) }\end{array}$ \\
\hline & $285 \mathrm{~nm}$ & (Freed \& Doehring 2005) \\
\hline & $290 \mathrm{~nm}$ & (Pradhan et al. 2012) \\
\hline \multirow{5}{*}{ Diameter $(D)$} & $1 \mathrm{~nm}$ & (Charvolin \& Sadoc 2012) \\
\hline & $1.4 \mathrm{~nm}$ & (Freed \& Doehring 2005) \\
\hline & $1.5 \mathrm{~nm}$ & $\begin{array}{l}\text { (Uzel \& Buehler 2009), } \\
\text { (Pradhan et al. 2012), (Parry } \\
\text { 1988), (Miller 1984), (Sun et } \\
\text { al. 2001) }\end{array}$ \\
\hline & $1.09 \mathrm{~nm}$ (dry) & $\begin{array}{l}\text { (Lees 1981), (Fratzl et al. } \\
\text { 1993) }\end{array}$ \\
\hline & $1.42-1.5 \mathrm{~nm}$ (wet) & $\begin{array}{l}\text { (Lees 1981), (Fratzl et al. } \\
\text { 1993) }\end{array}$ \\
\hline \multirow{4}{*}{$\operatorname{Pitch}(p)$} & $8 \mathrm{~nm}$ & $\begin{array}{l}\text { (Bozec \& Horton 2005), (Sun } \\
\text { et al. 2001) }\end{array}$ \\
\hline & $8.58 \mathrm{~nm}$ & $\begin{array}{l}\text { (Bhattacharjee \& Bansal } \\
\text { 2005) }\end{array}$ \\
\hline & $8.55 \mathrm{~nm}$ & (Beck \& Brodsky 1998), \\
\hline & $8.68 \mathrm{~nm}$ & (Fraser et al. 1983) \\
\hline
\end{tabular}

The TC configuration consists of three similar helices having the same axis, but differ in the starting angle along the axis. The center line $\left(\vec{r}_{s}\right)$ of one helix can be obtained by Neurkich mathematical formulation (Neukirch et al. 2008):

$\vec{r}_{s}=\left\{\begin{array}{c}+\frac{D}{2} \sin w(s) \\ -\frac{D}{2} \cos w(s) \\ s \cos \theta\end{array}\right.$, 
where $w(s)$ is the equatorial angle:

$w(s)=s \frac{\sin \theta}{D / 2}$,

$s$ the curvilinear abscissa, $D$ the super helical diameter, $\theta$ the helical angle, and $\vec{r}_{s}$ spans a helix of a pitch:

$p=\frac{\pi D}{\tan \theta}$

The TC geometric model has a periodic symmetry along the longitudinal axis with a period $p$, which makes it possible to represent the entire model by a single pitch (Figure 1). The nonhelical ends of the molecule are modeled by cylindrical portions attached to the ends of the helices.

The geometric dimension variability found in the literature (Table 1) leads us to build a parameterized model. The latter has been generated by the design CATIA software in order to study the changing influence of the TC molecule diameter $(D)$, the spacing separating the alpha helices of the same TC molecule as well as the TC pitch $(p)$ on its mechanical behavior, Figure 2.

The geometric parameters of the first tested TC molecule were: $L$ equal to $300 \mathrm{~nm}, p$ to 6.5 $\mathrm{nm}, D$ to $1.5 \mathrm{~nm}$ and $d$ to $0.45 \mathrm{~nm}$. The obtained results corresponding to this molecule were used to validate the current finite element model by comparing them against results of other published studies found in the literature.

In order to study the variation effect of the TC diameter, the geometric parameters of the various tested molecules were (Figure 2a):

- $\quad$ The fixed parameters: $L=300 \mathrm{~nm}, p=6.5 \mathrm{~nm}, d=0.45 \mathrm{~nm}$;

- The variable parameter $D$ that took respectively the following values: $1,1.2,1.3$ and $1.5 \mathrm{~nm}$.

While the following geometric parameters of the various tested molecules (Figure $2 b$ ) were used to study the spacing effect between the alpha helices:

- The fixed parameters: $L=300 \mathrm{~nm}, p=6.5 \mathrm{~nm}, D=1.5 \mathrm{~nm}$;

- The variable parameter $d$ that took respectively the following values: $0.25,0.35,0.45$ and $0.55 \mathrm{~nm}$. 


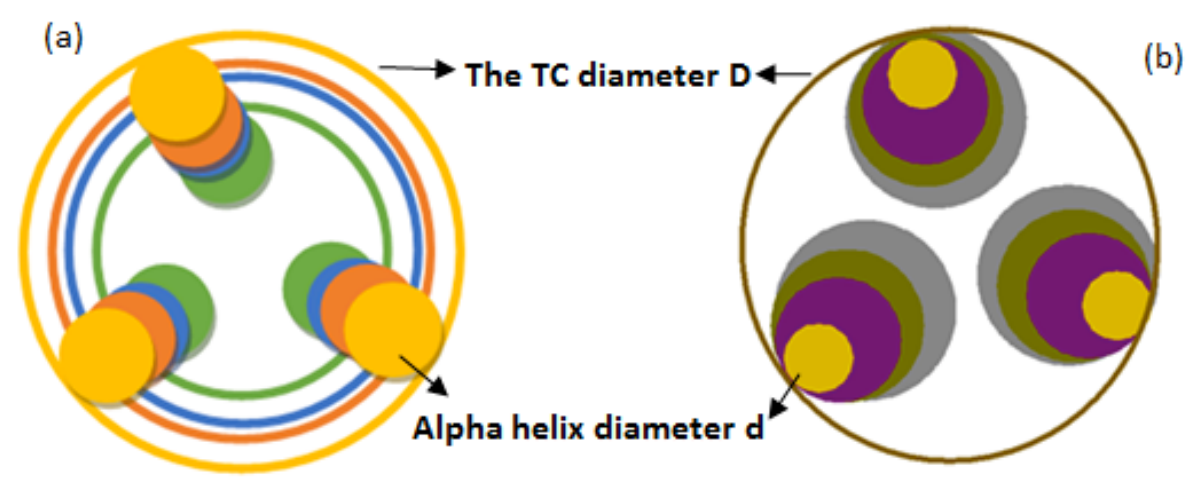

Figure 2: View from above of different superposed TC molecule that have been tested (the same color for the three alpha helices of each TC molecule is used): (a) TC models tested to study the molecule diameter effect; (b) TC models tested to study the alpha helices spacing effect having the same TC diameter molecule $(D)$ equal to $1.5 \mathrm{~nm}$.

In order to study the pitch variation effect, the geometric parameters of the various tested molecules were the following (Figure 2b):

- The fixed parameters: $L=300 \mathrm{~nm}, D=1.5 \mathrm{~nm}, d=0.45 \mathrm{~nm}$;

- The variable parameter $p$ with value $6.5,7.5,8.6$ and $9.6 \mathrm{~nm}$.

\section{FE model generation}

The geometric TC model was exported from CATIA software into ABAQUS finite element analysis software.

Boundary conditions (BC) were defined as: one end is fixed while the other end is restrained with displacement along the molecular axis. All the tested molecules were stretched with an imposed displacement of $50 \%$ of their pitch $(p)$, Figure 3 .

Concerning the mesh, a linear hexahedral element, C3D8, with eight nodes and six faces, was used to discretize the geometric model. 


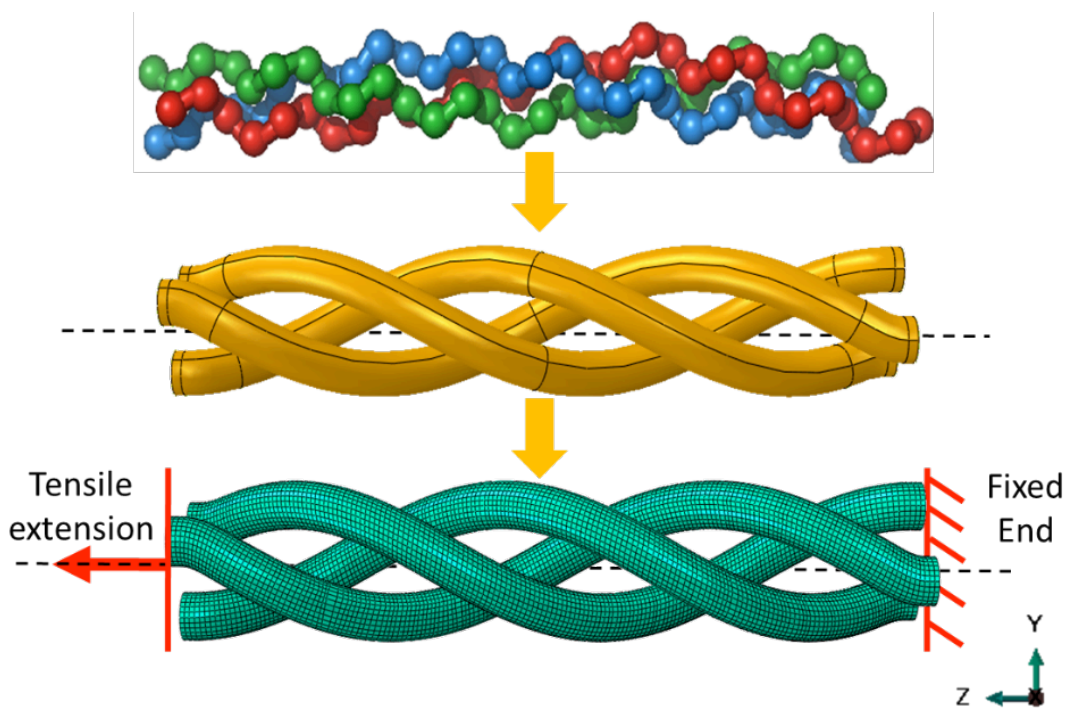

Figure 3: FE TC modeling: From molecular structure to a FE model discretization with boundary conditions.

Alpha helix properties were integrated into the FE model, for which the elastic properties for the entropic response (small extension) were: Young's modulus $(E)$ equal to $2.5 \mathrm{GPa}$ (Buehler $\&$ Keten 2008) and Poisson ratio (v) of 0.3 (Tang et al. 2006). The contact between the different helices was included into ABAQUS as a surface-to-surface contact with a small sliding formulation. A frictionless tangential behavior and a hard normal behavior were observed.

\section{Analysis of nanomechanical behavior}

Simulation using FEM allows to avoiding costly and harmful tests as well as detailed visualization of where bending or twisting structures occur. Furthermore, the stress and the strain distribution can be estimated when forces are applied. In this work, the stress $(\sigma)$ denotes the amount of the internal forces that arise from the object deformation, and the strain $(\varepsilon)$ corresponds to the size of the object change when a force or a displacement is imposed:

$$
\sigma=E * \varepsilon=\frac{F}{S}
$$

where $E$ is the Young's modulus, $F$ the applied force and $S$ the section of the simulated structure. 
Young's modulus $(E)$ correlates with both the stress and the strain (Equation 4), and measures the material stiffness. Within the elastic range, $E$ is defined as the stress derivative while respecting the strain that is evaluated at the strain $(\varepsilon)$ and can be obtained by force normalization by the cross sectional area of the simulated structure.

\section{Results and Discussion}

The current work has been carried out with FEM simulating a stretching TC molecule. The characteristic behavior of the force-reduced extension is shown in Figure 4. The aim was to conduct a quantitative analysis of the TC mechanical behavior.

The nanoscopic-reduced extension is defined by $x / L$, where $x$ is the molecular extension along the molecular axis as a response of the applied displacement, and $L$ the model length. In our case, it was adopted $L=p$.

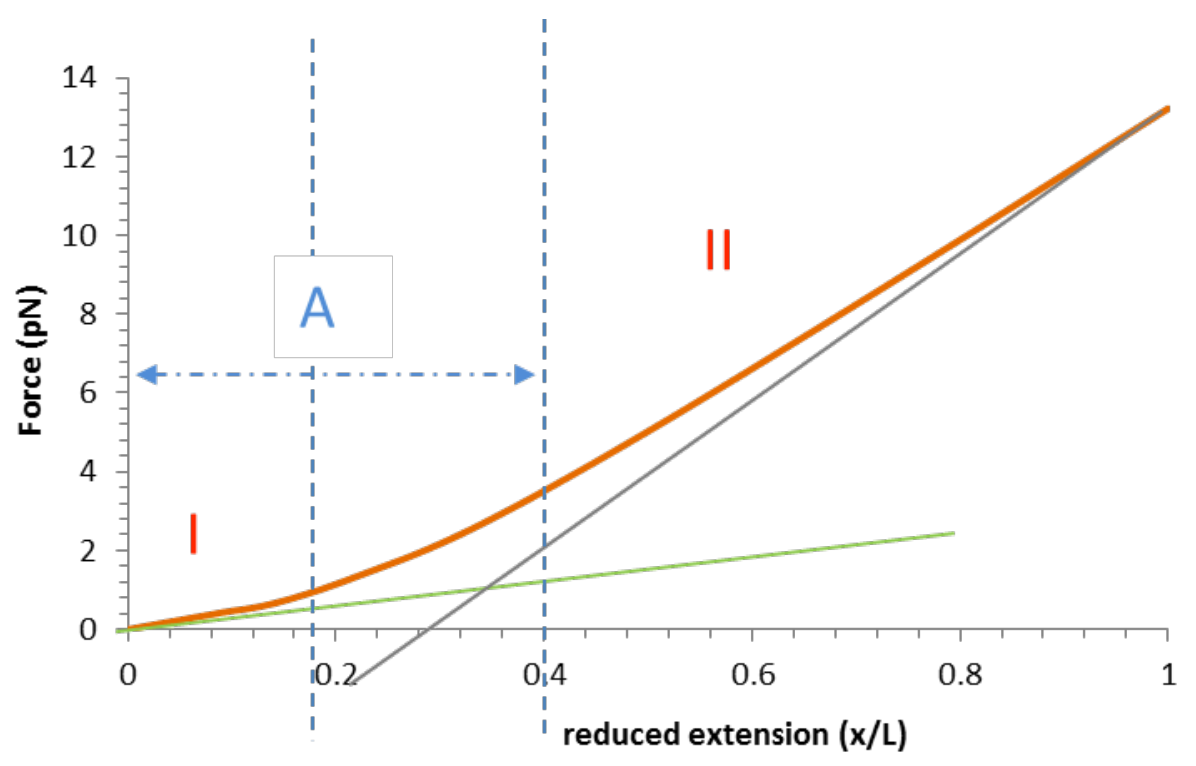

Figure 4: The force-reduced extension curve of the stretching FE simulation results of a single TC molecule, focusing on the entropic response (small force $F<14 \mathrm{pN}$ ). The molecule length $(L)$ was equal to $300 \mathrm{~nm}$, the molecule diameter to $1.5 \mathrm{~nm}$, and the alpha helix diameter $(d)$ to $0.45 \mathrm{~nm}$. The imposed displacement was of $50 \%$ of the pitch $(p)$. The alpha helix young modulus $(E)$ was equal to $2.5 \mathrm{GPa}$. 
The TC force-reduced extension plot (the orange line in Figure 4) depicts two zones I and II. This curve shows a continuous stiffening starting from a small tangent modulus until reaching $20 \%$ extension, with the modulus increasing continuously. The first zone of the curve is characterized by a low force and is called "toe region". This latter represents the stretching of parallel alpha helices and is followed by the "heel region", where the slope increases due to the establishment of contact between the different alpha helices. The force in the second zone is quite small not exceeding a few $\mathrm{pN}$. The second regime is characterized by the molecule end rotation to accommodate the uncoiling mechanisms of the triple helix. The characteristic triple helical structure provides the structural basis for this continuous stiffening behavior.
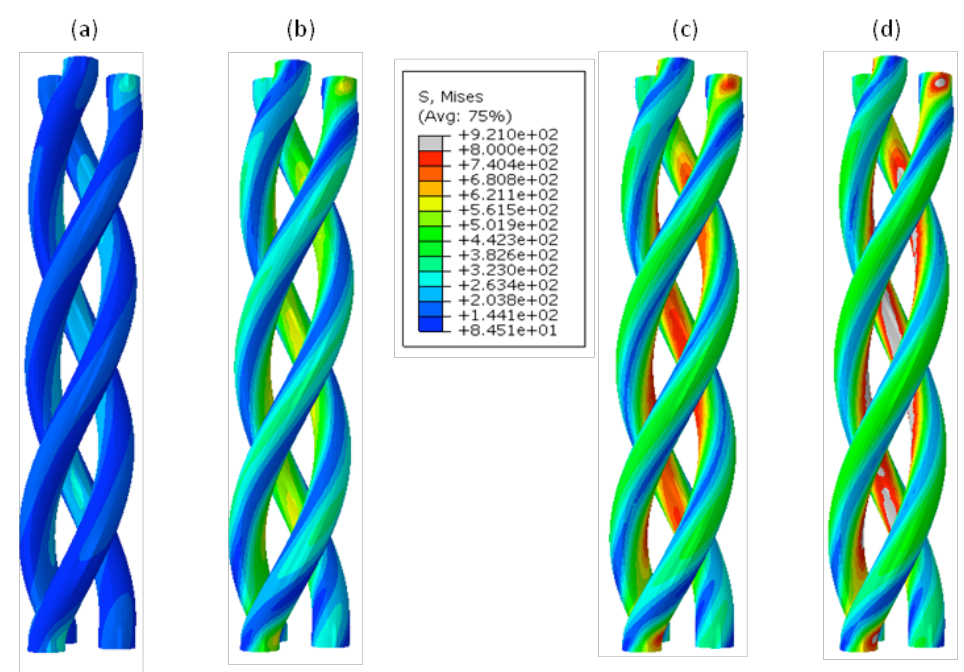

Figure 5: von Mises contour of different increments (a); there is no contact between the three alpha helices: each helix is just constrained by the imposed tensile displacement (b); the beginning of the establishment contact between the three helices; (c) the contact surface increases; and (d) the contact surface reaches its maximum relative to the imposed displacement.

The four Von Mises stress distributions showed in Figure 5 are related to four configurations of the TC molecule at different increments. The two first configurations (Figures $5 \mathrm{a}$ and $\mathrm{b}$ ) correspond to two increments located in the zone I of the curve of Figure 4. In other hand, the two second configurations (Figures $5 \mathrm{c}$ and d) correspond to two increments located in zone II of the curve of Figure 4. Figure 5 illustrates the Von Mises stress distributions that confirm what happens at the molecule level when the slope of the curve force-reduced extension (Figure 4) is changed. As long as the force applied increases, the Von Mises stress concentration increases, mainly in the contact zone between the different alpha helices. 
The obtained TC force-reduced extension curve (in orange in Figure 4) has the same shape of that found by Buehler and Keten (Buehler \& Keten 2008), Sun and co-authors (Sun et al. 2002) and Bozec and Horton (Bozec \& Horton 2005). The TC molecule geometric parameters are similar to those tested in the current work. Buehler and Keten (Buehler \& Keten 2008) applied a tensile force on a TC molecule using the molecular dynamic method. The forcestrain curve deducted has a bilinear fit that marks two deformation regimes: small and large deformation. An initial linear elastic regime is followed by the second regime at large strains that can get beyond $30-35 \%$ of strain. Buehler and Keten suggested that the first regime is characterized by a TC molecule uncoiling, and the second regime is associated with a larger modulus due to stretching of covalent bonds. Using optical tweezers, Sun and collaborators (Sun et al. 2002) found a force-extension curve presenting also two zones: a toe region in the small strain and a heel region in the large one. This curve was fitted to Marco and Siggia entropic elasticity model called worm like chain WLC model (Marko \& Siggia 1995).

Figure 6 shows a quantitative comparison until 40\% of reduced extension (zone A in Figure 4) compared to experiments preformed using optical tweezers (Sun et al. 2002), molecular dynamic MD simulation (Buehler \& Wong 2007) and worm-like chain (WLC) model estimation (Bustamante et al. 1994; Marko \& Siggia 1995). The plot depicts a very good consistency among the findings.

Figure 7 shows the force-reduced extension curve for different tested TC molecule diameters $(D)$. By increasing the hydration degree, the TC molecule diameter increases and the slopes of the two regions of the different curves decrease. Consequently, the elastic properties deteriorate when the hydration degree increases (Figure 7). After fixing the TC diameter equal to $1.5 \mathrm{~nm}$, the following tests were aimed to determine the spacing effect between the three helices of the molecule with variation of the alpha helix diameter.

Figure 8 illustrates the force-reduced extension response in the case of a TC molecule under tensile loading. The results were obtained using different spacing values $(d)$ between alpha helices of the same TC molecule. 


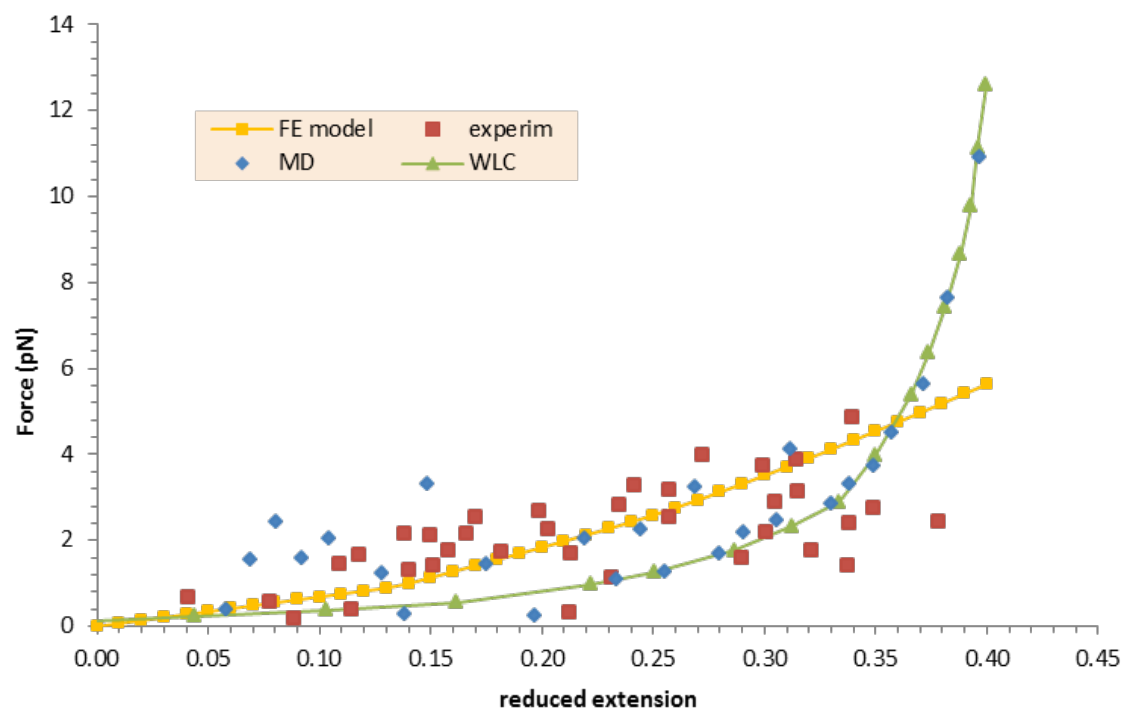

Figure 6: The force-reduced extension curve of a single TC results obtained with the finite element simulation. This plot also depicts results achieved for TC molecules with similar geometric parameters using molecular dynamic (MD) simulation, experimental tests and worm-like chain (WLC) elasticity model (theoretical model).
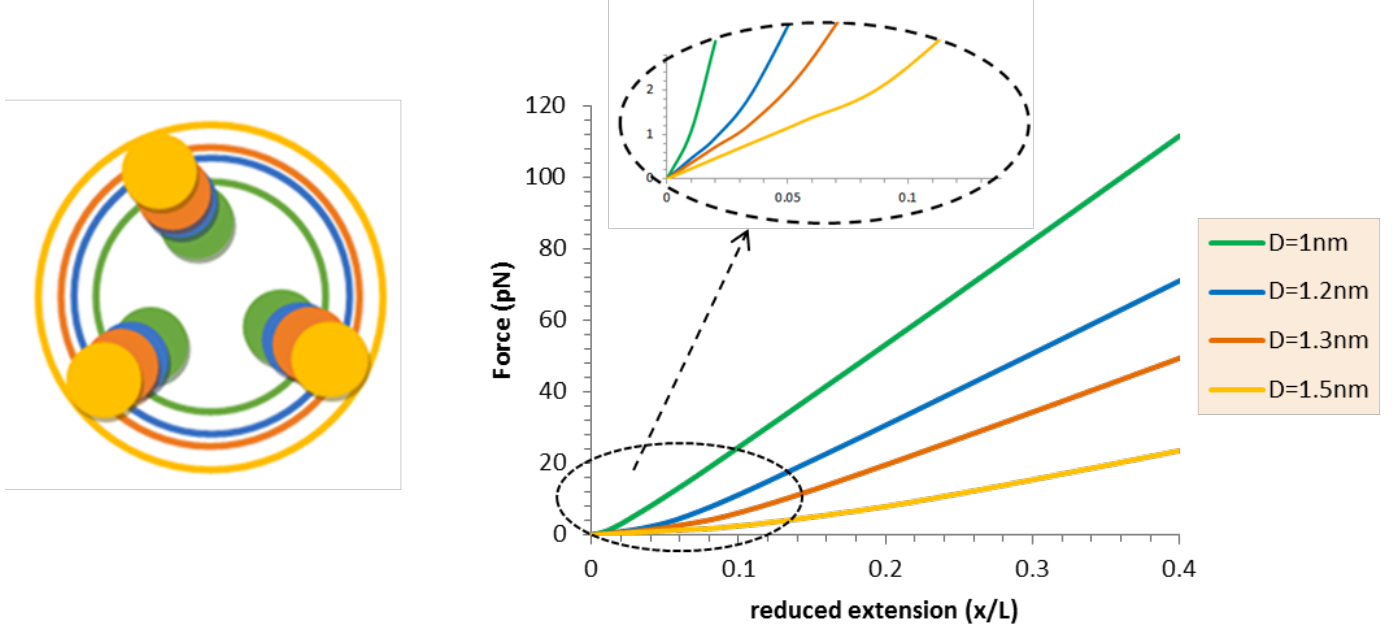

Figure 7: Force-reduced extension curve for different tested TC molecule diameters $(D)$. 

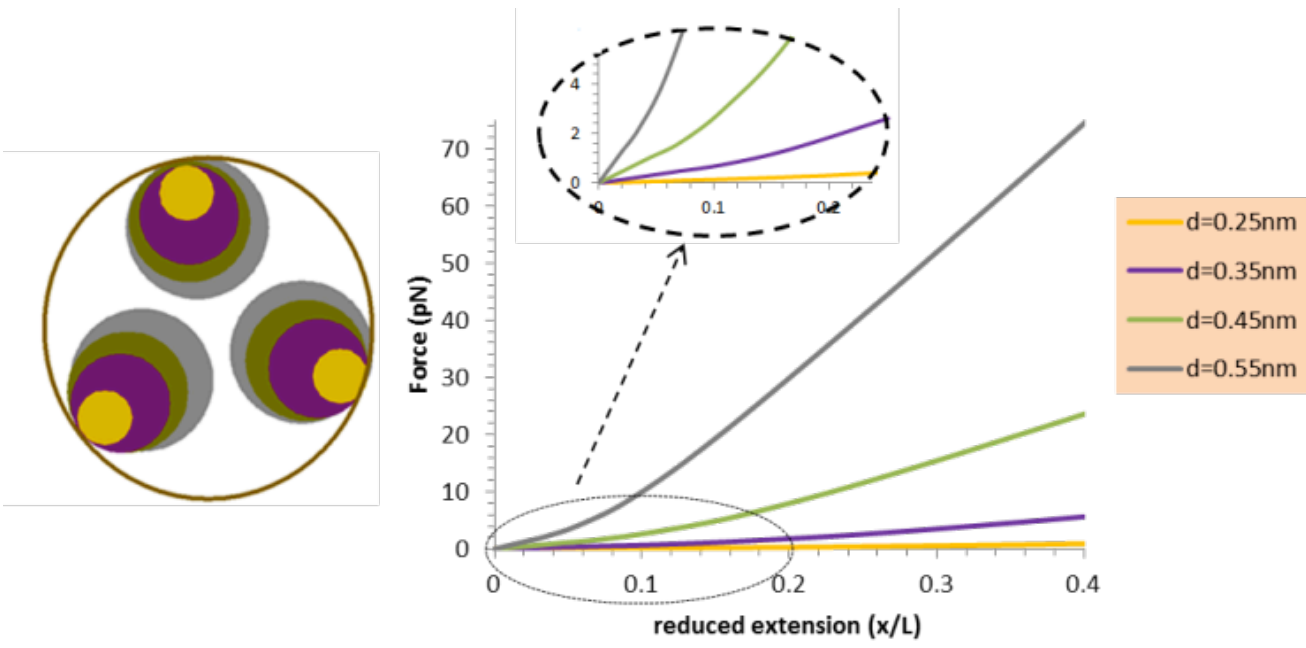

Figure 8: Force-reduced extension curve for the different tested spacing values $(d)$ between the different alpha helices of the same TC molecule.

The obtained results using the FE modeling show that the nature of the helix alpha (hydrated or dehydrated), which is modeled in this study by the changing of each alpha helix diameter, has an important effect on the mechanical behavior of TC molecules (Figure 8). The TC molecule elastic properties improve by increasing the hydration degree. Subsequently, Young's modulus increases when the space between the alpha helices decreases.

Figure 9 represents the force-reduced extension curve for different tested TC molecule pitch $(p)$.

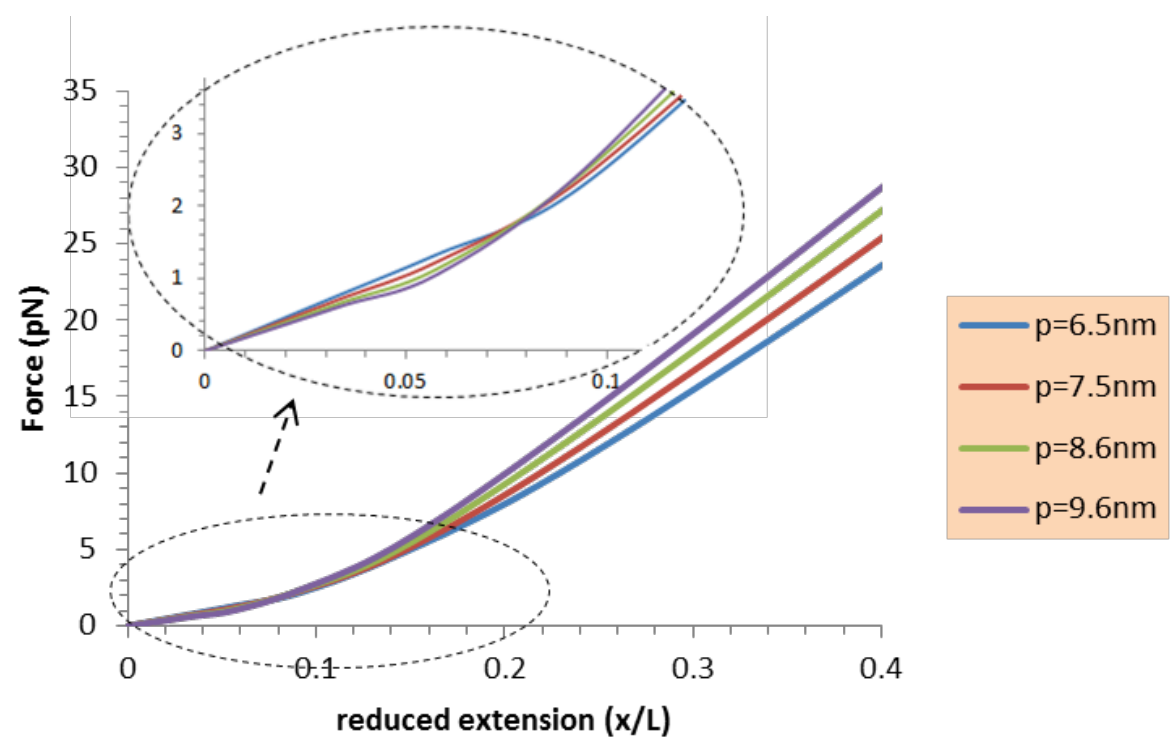

Figure 9: Force-reduced extension curve for different tested TC molecule pitch $(p)$. 
The finite element simulations (Figure 9) revealed that the pitch variation has a double effect on the mechanical properties of the TC molecule. For the small strain, the smaller pitch is the stiffer molecule. For the large strain, the pitch length impact is reversed: the small pitch is the more flexible molecule. Shoulders and Raines (Shoulders \& Raines 2010) investigated the influence of the atomic collagenous sequence on the value of the TC pitch. The poor proline structure has a longer pitch than the rich proline structure. It is then necessary to investigate the influence of the pitch length molecule on its mechanical properties. It is noticed that Buehler and Ackbarow ((Buehler 2006), (Buehler \& Ackbarow 2007)) and Lorenzo and Caffarena (Lorenzo \& Caffarena 2005) carried out their studies on proline rich molecules.

\section{Conclusion}

The aim of this work was to study the mechanical behavior of a TC molecule type I using FEM. A novel 3D FE model was proposed in order to describe the mechanical behavior of the coiled coil collagen formed by three alpha helices on the entropic regime.

During this study, a parametric model giving the variation impact of one of the geometric parameters on the mechanical behavior was built. The obtained result of a TC molecule tensile test among the molecular axis was compared with an entropic response of an experimental, a molecular dynamic and a theoretical study. The approached results allowed to validate the built FE model.

A series of FE models were carried out to investigate the effect of the TC diameter variation. The elastic properties of the dehydrated molecule are greater than those of the hydrated molecule. Another series of FE models were conducted to investigate the influence of the spacing between helices of the same molecule. Hence, these tests predicted that the spacing variation has a significant influence on the molecule stiffness. A last series of FE models were used to examine the impact of the pitch variation on the TC molecule mechanical behavior. In the small strain, the poor proline molecule is less stiff than the rich proline molecule. In the large strain, this behavior is reversed and the poor proline molecule is stiffer than the rich proline one.

In the future, additional tests will be performed to study the influence of the mechanical properties variation of the alpha helix. To pursuit this qualitative study, a quantitative study 
will also be conducted. Other than the elastic behavior, the proposed model can be used to study the viscoelastic and the fracture behavior of the TC molecule. In order to establish the remaining mechanical behavior beyond the entropic response, some improvements on the FE model must be made. The current study has been carried out using the alpha helix Young's modulus corresponding to the small strain. Nevertheless, the use of large strain Young's modulus can be also investigated. In addition, it will be interesting to improve the geometry of the model taking into account the cross-links between alpha helices.

\section{Conflict of Interest}

The authors declare that they have no conflict of interest.

\section{Acknowledgment}

João Manuel R.S. Tavares gratefully acknowledges the funding of Project NORTE-01-0145FEDER-000022 - SciTech - Science and Technology for Competitive and Sustainable Industries, cofinanced by "Programa Operacional Regional do Norte" (NORTE2020), through "Fundo Europeu de Desenvolvimento Regional" (FEDER).

\section{References}

Barkaoui, A., Chamekh, A., et al., 2014. Multiscale approach including microfibril scale to assess elastic constants of cortical bone based on neural network computation and homogenization method. International journal for numerical methods in biomedical engineering, 30(3), pp.318-38. Available at: http://www.ncbi.nlm.nih.gov/pubmed/24123969.

Barkaoui, A. \& Hambli, R., 2011. Finite element 3D modeling of mechanical behavior of mineralized collagen microfibrils. Journal of applied biomaterials \& biomechanics : $J A B B, 9(3)$, pp.199-205. Available at: http://arxiv.org/abs/1112.0984\nhttp://www.ncbi.nlm.nih.gov/pubmed/22139755.

Barkaoui, A., Hambli, R. \& Tavares, J.M.R.S., 2014. Effect of material and structural factors on fracture behaviour of mineralised collagen microfibril using finite element simulation. 
Computer methods in biomechanics and biomedical engineering, 5842(March 2015), pp.1-10. Available at: http://dx.doi.org/10.1080/10255842.2014.883601.

Beck, K. \& Brodsky, B., 1998. Supercoiled Protein Motifs: The Collagen Triple-Helix and the a-Helical Coiled Coil. Journal of Structural Biology, 122(1-2), pp.17-29.

Bella, J. et al., 1994. Crystal and molecular structure of a collagen-like peptide at 1.9 A resolution. Science, 266(5182), pp.75-81.

Bhattacharjee, A. \& Bansal, M., 2005. Collagen structure: the Madras triple helix and the current scenario. IUBMB life, 57(3), pp.161-172.

Bozec, L. \& Horton, M., 2005. Topography and mechanical properties of single molecules of type I collagen using atomic force microscopy. Biophysical journal, 88(6), pp.42234231 .

Buehler, M.J., 2006. Atomistic and continuum modeling of mechanical properties of collagen: Elasticity, fracture, and self-assembly. Journal of Materials Research, 21, pp.19471961.

Buehler, M.J. \& Ackbarow, T., 2007. Fracture mechanics of protein materials. Materials Today, 10(9), pp.46-58.

Buehler, M.J. \& Keten, S., 2008. Elasticity, strength and resilience: A comparative study on mechanical signatures of $\alpha$-Helix, $\beta$-sheet and tropocollagen domains. Nano Research, 1(1), pp.63-71.

Buehler, M.J. \& Wong, S.Y., 2007. Entropic elasticity controls nanomechanics of single tropocollagen molecules. J biomech, 93(1), pp.37-43.

Bustamante, C. et al., 1994. Entropic elasticity of lambda-phage DNA. Science (New York, N.Y.), 265(1994), pp.1599-1600.

Charvolin, J. \& Sadoc, J.-F., 2012. About collagen, a tribute to Yves Bouligand. Interface focus, 2(5), pp.567-74.

Van Duin, A.C.T. et al., 2001. ReaxFF: A reactive force field for hydrocarbons. Journal of Physical Chemistry A, 105(41), pp.9396-9409.

Fraser, R.D.B. et al., 1983. Molecular conformation and packing in collagen fibrils. Journal of Molecular Biology, 167(2), pp.497-521. 
Fratzl, P., 2008. Collagen: Structure and mechanics, an introduction. In Collagen: Structure and Mechanics. pp. 1-13.

Fratzl, P., Fratzl-Zelman, N. \& Klaushofer, K., 1993. Collagen packing and mineralization. An x-ray scattering investigation of turkey leg tendon. Biophysical journal, 64(1), pp.260-6.

Freed, A.D. \& Doehring, T.C., 2005. Elastic model for crimped collagen fibrils. Journal of biomechanical engineering, 127, pp.587-593.

Gautieri, A. et al., 2012. Viscoelastic properties of model segments of collagen molecules. Matrix Biology, 31(2), pp.141-149.

Hulmes, D.J.S., 2008. Collagen diversity, synthesis and assembly. In Collagen: Structure and Mechanics. pp. 15-47.

Kadler, K.E. et al., 1996. Collagen fibril formation. Biochem J, 316 ( Pt 1, pp.1-11.

Kadler, K.E. et al., 2007. Collagens at a glance. Journal of Cell Science, 120(12), pp.19551958.

Lees, S., 1981. A mixed packing model for bone collagen. Calcified tissue international, 33(6), pp.591-602.

Lorenzo, A.C. \& Caffarena, E.R., 2005. Elastic properties, Young's modulus determination and structural stability of the tropocollagen molecule: A computational study by steered molecular dynamics. Journal of Biomechanics, 38(7), pp.1527-1533.

MacKerell, A.D. et al., 1998. All-Atom Empirical Potential for Molecular Modeling and Dynamics Studies of Proteins †. The Journal of Physical Chemistry B, 102(18), pp.3586-3616.

Marko, J.F. \& Siggia, E.D., 1995. Stretching DNA. Macromolecules, 28(26), pp.8759-8770.

Miller, A., 1984. Collagen: The Organic Matrix of Bone. Philosophical Transactions of the Royal Society of London. Series B, Biological Sciences, 304(1121), pp.455-477.

Neukirch, S., Goriely, A. \& Hausrath, A.C., 2008. Chirality of coiled coils: Elasticity matters. Physical Review Letters, 100(3).

Orgel, J.P.R.O. et al., 2001. The in situ supermolecular structure of type I collagen. Structure, 9(11), pp.1061-1069. 
Parry, D.A.D., 1988. The molecular fibrillar structure of collagen and its relationship to the mechanical properties of connective tissue. Biophysical Chemistry, 29(1-2), pp.195-209.

Pauling, L. \& Corey, R.B., 1953. A Proposed Structure For The Nucleic Acids. Proceedings of the National Academy of Sciences of the United States of America, 39(2), pp.84-97.

Pradhan, S.M., Katti, K.S. \& Katti, D.R., 2012. Structural hierarchy controls deformation behavior of collagen. Biomacromolecules, 13(8), pp.2562-2569.

Ramachandran, G.N. \& Kartha, G., 2006. Structure of Collagen. 1954, 1955. The National medical journal of India, 19(6), pp.348-352.

van der Rest, M. \& Garrone, R., 1991. Collagen family of proteins. The FASEB journal : official publication of the Federation of American Societies for Experimental Biology, 5(13), pp.2814-2823.

Saini, K. \& Kumar, N., 2015. Mechanical response of collagen molecule under hydrostatic compression. Materials Science \& Engineering C, 49, pp.720-726.

Sasaki, N. \& Odajima, S., 1996. Stress-strain curve and young's modulus of a collagen molecule as determined by the X-ray diffraction technique. Journal of Biomechanics, 29(5), pp.655-658.

Shoulders, M.D. \& Raines, R.T., 2010. Collagen Structure and Stability. Annu Rev Biochem, 78, pp.929-958.

Sun, Y.L. et al., 2002. Direct quantification of the flexibility of type I collagen monomer. Biochemical and Biophysical Research Communications, 295(2), pp.382-386.

Sun, Y.L., Luo, Z.P. \& An, K.N., 2001. Stretching short biopolymers using optical tweezers. Biochemical and biophysical research communications, 286(4), pp.826-30.

Tang, Y. et al., 2006. A Finite Element Framework for Studying the Mechanical Response of Macromolecules: Application to the Gating of the Mechanosensitive Channel MscL. Biophysical Journal, 91(4), pp.1248-1263.

Uzel, S.G.M. \& Buehler, M.J., 2009. Nanomechanical sequencing of collagen: tropocollagen features heterogeneous elastic properties at the nanoscale. Integrative biology: quantitative biosciences from nano to macro, 1(7), pp.452-459.

in 't Veld, P.J. \& Stevens, M.J., 2008. Simulation of the mechanical strength of a single 
collagen molecule. Biophysical journal, 95(1), pp.33-9.

Vercher, A. et al., 2014. Homogenized stiffness matrices for mineralized collagen fibrils and lamellar bone using unit cell finite element models. Biomechanics and Modeling in Mechanobiology, 13(2), pp.437-449.

Vercher-Martinez, A. et al., 2015. Influence of the mineral staggering on the elastic properties of the mineralized collagen fibril in lamellar bone. Journal of the Mechanical Behavior of Biomedical Materials, 42, pp.243-256. 